\title{
How the punctuality of the Shinkansen has been achieved
}

\author{
N. Tomii \\ Chiba Institute of Technology, Japan
}

\begin{abstract}
The high speed railway line in Japan began operation in 1964. The high speed railway is called the Shinkansen and is known for its safety and reliability. In addition, the Shinkansen is well known for punctuality. As a matter of fact, the average delay of trains is less than one minutes every year. The Shinkansen runs along dedicated lines, which seem to be advantageous in keeping punctuality. However, there are lots of disadvantages as well. For example, although traffic is very dense, resources are not abundant. In some Shinkansen lines, trains go directly through conventional railway lines and the Shinkansen is easily influenced by the disruption of those lines. Punctuality of the Shinkansen is supported by hardware, software and humanware. In this paper, we first introduce a brief history of the Shinkansen and then focus on humanware, which makes the punctuality possible.
\end{abstract}

Keywords: high speed trains, punctuality, rescheduling, Shinkansen.

\section{Introduction}

In 1964, a high speed railway line opened in Japan. The new line connects Tokyo, the capitol, and Osaka, the second largest city located $600 \mathrm{~km}$ away. The maximum speed of trains was $210 \mathrm{~km} / \mathrm{h}$, which was almost twice that of other trains in those days and the travelling time between these two cities was halved to only three hours and ten minutes.

The new high-speed line was called the Shinkansen and it had a great impact not only on railways in Japan, but also on railways worldwide.

From that time on, the Shinkansen was extended to other areas of Japan and the length of Shinkansen lines is about 2,200 km at present.

We may well say that characteristics of the Shinkansen are very dense traffic, very high safety and very high punctuality. 
In the Tokaido Shinkansen line, the total number of trains a day is 323 at present. As a matter of fact, at Tokyo station, you can see trains departing every several minutes.

The Shinkansen is proud of ultimate safety with evidence that no passengers in trains were killed or injured for 46 years since the Shinkansen began its operation.

Another important characteristic is punctuality. In the fiscal year 2008, the average delay of trains in the Tokaido Shinkansen was just 0.6 minutes, namely 36 seconds. People might think this punctuality is achieved thanks to abundant resources, such as extra train-sets, extra crews, lots of tracks at stations, etc. However, this is not true. As described later, resources are not abundant. The punctuality of the Shinkansen is achieved by hardware, software and humanware.

As for the hardware, efficiency and reliability of signalling systems, electrical power transmission, tracks and rolling stocks play quite an important role in keeping punctuality.

As for the software, the Shinkansen is equipped with various kinds of computer systems. To name a few, route control systems, operation management systems, track maintenance management systems, rolling stock maintenance management systems, etc, which are indispensable in keeping the punctuality of the Shinkansen.

Humanware, however, is very important as well. In this paper, the importance of humanware to keep punctuality is focused upon.

\section{Brief history of the Shinkansen}

An outline of the Shinkansen network is given in Table 1 and Figure 1. In Japan, each line is given a line name such as Tokaido, Shinkansen and Sanyo Shinkansen and so on.

Table 1: $\quad$ Outline of the Shinkansen network.

\begin{tabular}{c|c|c|c|c}
\hline Line & From & To & Distance & Remark \\
\hline \hline Tokaido & Tokyo & Osaka & $515.4 \mathrm{~km}$ & \\
\hline Sanyo & Osaka & Hakata & $553.7 \mathrm{~km}$ & Trains start from Tokyo. \\
\hline Tohoku & Tokyo & Hachinohe & $593.1 \mathrm{~km}$ & $\begin{array}{c}\text { Extended to Aomori } \\
(2010 / 12)\end{array}$ \\
\hline Joetsu & Omiya & Niigata & $269.5 \mathrm{~km}$ & Trains start from Tokyo. \\
\hline Hokuriku & Takasaki & Nagano & $117.4 \mathrm{~km}$ & Trains start from Tokyo. \\
\hline Akita & Morioka & Akita & $127.3 \mathrm{~km}$ & Trains start from Tokyo. \\
\hline Yamagata & Fukushima & Shinjo & $148.6 \mathrm{~km}$ & Trains start from Tokyo. \\
\hline Kyushu & Yatsushiro & Kagoshima & $126.8 \mathrm{~km}$ & $\begin{array}{c}\text { Extended to Hakata } \\
(2011 / 3)\end{array}$ \\
\hline
\end{tabular}




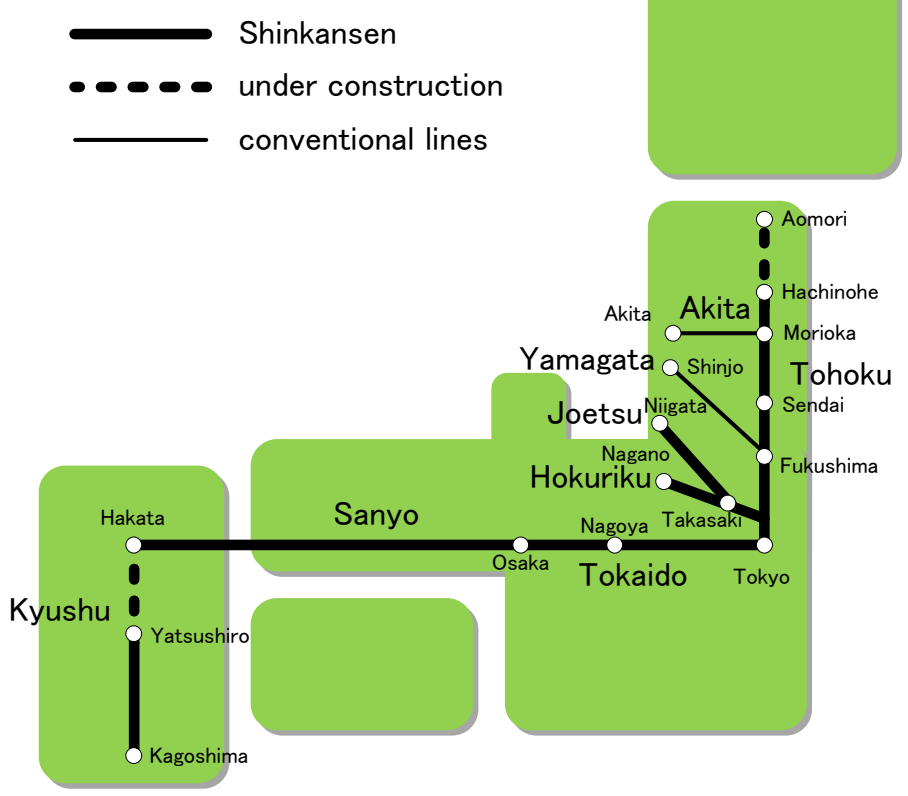

Figure 1: $\quad$ The Shinkansen network.

Although the Tokaido Shinkansen belongs to JR (Japanese Railways) Central Co. Ltd. and the Sanyo Shinkansen belongs to JR West Co. Ltd, trains on these two Shinkansen lines are operated jointly by these two companies because trains on the Tokaido Shinkansen go directly to the Sanyo Shinkansen and vice versa. The Tohoku, Joetsu, Nagano, Akita and Yamagata Shinkansens are managed by JR East Co. Ltd and the Kyushu Shinkansen is managed by JR Kyushu Co. Ltd.

The maximum speed of the Shinkansen is $300 \mathrm{~km} / \mathrm{h}$ at present, and an increase of the speed is in the planning stage.

Since its start of operation, the Shinkansen has taken a lot of passengers from airplanes. As a result, the flight service between Tokyo and Nagoya and between Tokyo and Sendai were given up after the Shinkansen began operation. At present, the Shinkansen keeps $60 \%$ of the market share between Tokyo and Akita and $81 \%$ market share between Tokyo and Osaka, for example [1, 2].

Figure 2 shows the annual transportation volume of the Shinkansen network and Figure 3 shows the market share of the Shinkansen for passenger transportation in fiscal year 2007 [3]. As you can see, the Shinkansen bears 82,825 million person kilometres, which is $6 \%$ of the market share of all over Japan.

The Shinkansen was designed with a brand new philosophy. It is totally different from railways in those days (railways other than the Shinkansen are called conventional railway lines in contrast with the Shinkansen). Major differences between the Shinkansen and conventional railway lines are: 


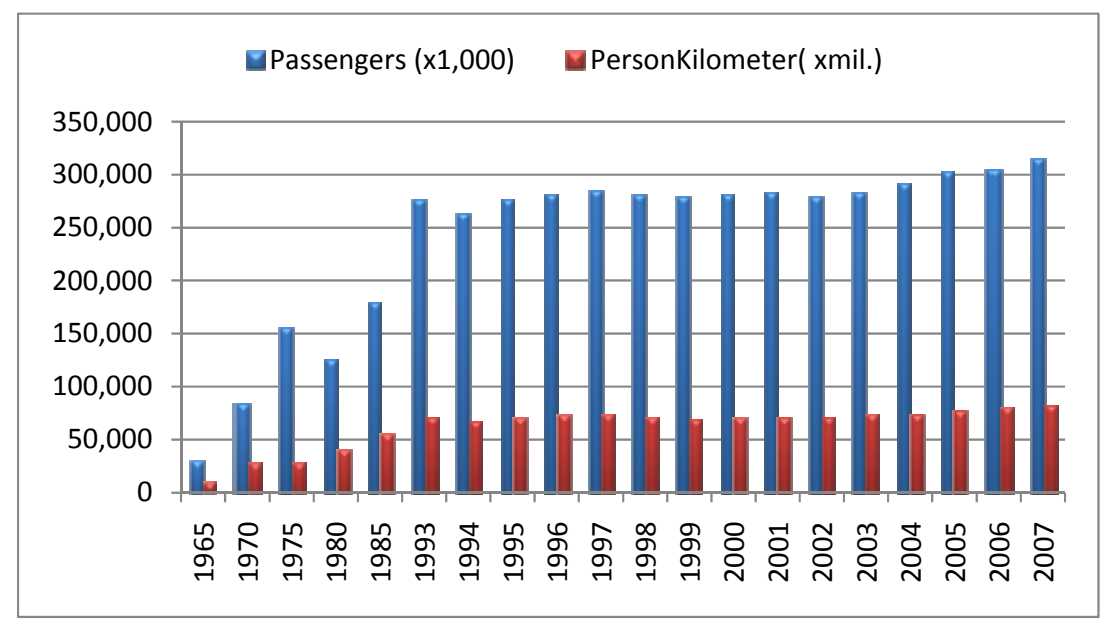

Figure 2: Annual transportation volume of the Shinkansen.

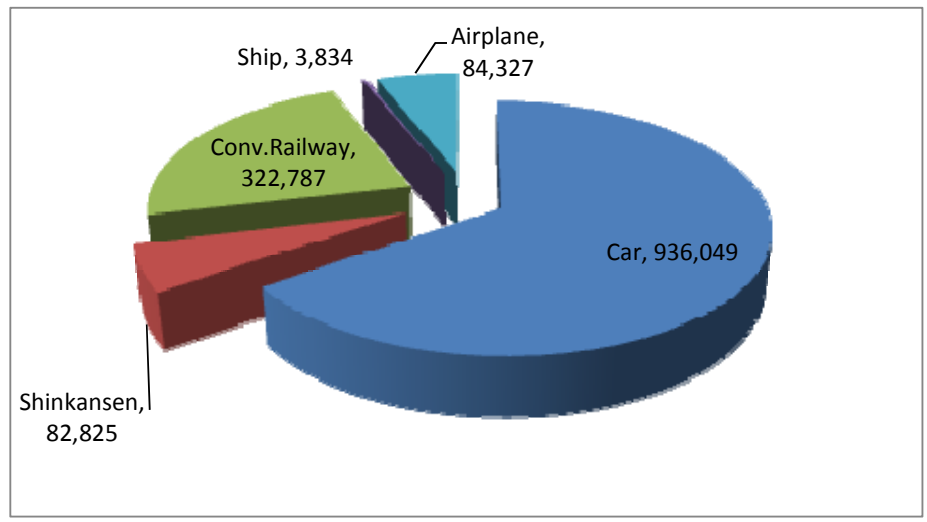

Figure 3: Market share of the Shinkansen.

1. The Shinkansen runs on dedicated lines, all of which were newly constructed.

2. The gauge is standard $(1435 \mathrm{~mm})$ whereas that of conventional railway lines is narrow $(1067 \mathrm{~mm})$.

3. Along all the lines, high fences are put to prevent public from approaching the tracks. There are no level crossings. These are established by special laws for the Shinkansen, which specify the rules about construction and operation of the Shinkansen.

4. Train schedules are rather simple compared with those of conventional railway lines. No freight trains are running and no night trains are running, for example. 
The Yamagata Shinkansen and the Akita Shinkansen are a bit different from other Shinkansens. The Yamagata and Akita Shinkansen lines are not regarded as Shinkansens from a legal point of view. Trains of the Tohoku Shinkansen go directly into these Shinkansen lines where trains other than the Shinkansen are also running. The gauges are standard (gauge was broadened so that the Shinkansen train-set can run when the Yamagata and Akita Shinkansens opened. In some part, lines are equipped with three rails so that both trains of the Shinkansen and trains of conventional railway lines can run) but the special laws about the Shinkansen are not applied. So, there are level crossings and no fences along the line etc. Trains are coupled and decoupled at the junction stations (Fukushima for the Yamagata Shinkansen and Morioka for the Akita Shinkansen).

One of the most remarkable characteristics of the Shinkansen is high punctuality. The average delay of the Tokaido Shinkansen is depicted in Figure 4[4]. In Japan, if a train is more than one minute behind the planned schedule, the train is considered to be "delayed" (this rule is the same in conventional railway lines). From Figure 4, we can observe that average delay of the Tokaido Shinkansen has been less than one minute for almost twenty years. The figures for the Tohoku and Joetsu Shinkansens are a bit larger than those of the Tokaido Shinkansen because as stated earlier, the punctuality of the Tohoku Shinkansen is easily influenced by the delay of trains in conventional railway lines. However, the figures are also less than one minutes every year recently.

\section{Disadvantages in keeping punctuality}

It may well be said that the Shinkansen is in an advantageous situation in keeping punctuality. In other words, special attention has been paid to prevent various kinds of disturbance from occurring.

In fact, collision with cars at level crossings which often happen in conventional railway lines never happen in the Shinkansen (except the Yamagata and Akita Shinkansens). People often commit suicide in railway lines but this seldom happen in the Shinkansen neither.

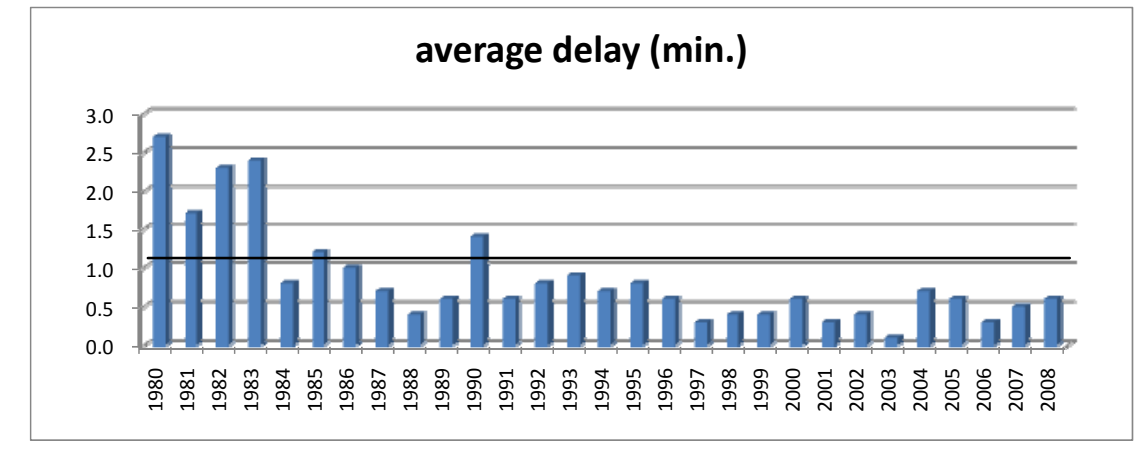

Figure 4: Average delay of the Tokaido Shinkansen. 


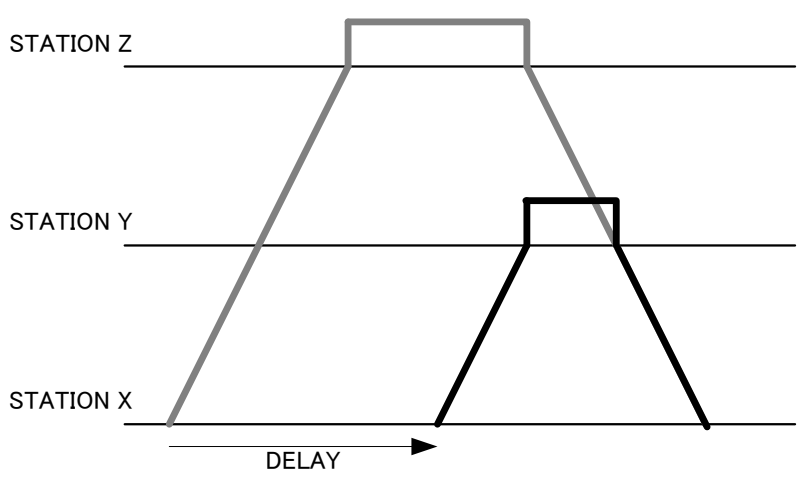

Figure 5: $\quad$ Partial cancellation of trains (grey: planned, black: result).

Still there are a lot of disadvantages as follows:

1. Trains are operated very densely. In the Tohoku and Joetsu Shinkansens, This means if a train is delayed, a lot of other trains are influenced. So, an extensive rescheduling is required.

2. It is difficult to use effective rescheduling methods. Cancelation of trains, which is an effective measure in rescheduling, is usually difficult to use in the Shinkansen. This is because trains run for a long distance and trains have seat reservations. For the similar reason, partial cancelation is never done in the Shinkansen. In conventional railway lines, partial cancelation of trains (see Figure 5) is usually done to absorb delays but this is never done in the Shinkansen because a lot of passengers are inconvenienced at Station Y.

Partial cancellation of trains is never done in the Shinkansen.

3. It is necessary to keep enough time at terminal stations for turning out, because cleaning inside trains and exchange of linen etc. are necessary. This means it is difficult to absorb delays at terminal stations.

4. Resources are not abundant. Basically, there are no stand-by train-sets and crews and there are not abundant tracks at stations. For example, Tokyo station of the Tokaido Shinkansen from which 14 trains per hour depart in the busiest time, has only six tracks. In Tokyo station of the Tohoku-Joetsu Shinkansen, there are only four tracks, from which eight trains per hour depart in the busiest time (It is all right to understand that there two Tokyo stations; one has six tracks and the other has four tracks). This becomes a serious constraint in rescheduling.

5. Natural disaster often happens and disrupts punctual train operation. In some part of the Tokaido Shinkansen, they sometimes have a severe snowfall in winter. Snowy weather itself is not a problem at all. However, if there is a snowfall, snow sticks to the surface of train-sets 
and these cause problems. That is, when the train-sets come to a rather warmer area, the snowballs fall down and hit ballast. Then the stone hit the train-set or houses along the Shinkansen line. So, trains are compelled to decrease running speed in snowy area so that snow does not stick to the train-set. In the Akita and Yamagata Shinkansens, on the other hand, trains run through regions where weather especially in winter is harsh and sometimes trains are delayed because they have to decrease the running speed.

6. Connection with trains in conventional railway lines is considered to be very important. The Shinkansen takes charge of long distance transportation, and timetables of conventional railways are made taking convenient connection with the Shinkansen into full account. This means, however, if trains are delayed in conventional railway lines, the Shinkansen trains have to wait although a limit of waiting time is prescribed a priori.

7. In the Yamagata and Akita Shinkansens, trains go directly to conventional railway lines. In conventional railway lines, trains other than the Shinkansen including freight trains are also running. The Shinkansen trains in these two lines are coupled or decoupled at junction stations as stated above, and this implies that a delay in these two lines is easily propagated to the Tohoku Shinkansen, the Joetsu Shinkansen and the Hokuriku Shinkansen because these Shinkansens share a track in some part.

8. Route control is done by computer systems (PRC: Programmed Route Control system) totally automatically. You may think this is advantageous in keeping punctuality. However, should a system-down occur, it might cause a serious problem. Reliability of the PRC is very high but the higher the reliability is, the less skilled dispatchers are in manual operation of signals. Although a system-down is very unlikely to happen, trains do not run on time if it really happens.

\section{Realizing the punctuality of the Shinkansen}

As described in detail in the previous section, there are a lot of disadvantages to achieve high punctuality in the Shinkansen. In this section, efforts and devices to concur the disadvantages are introduced.

1. As elaborate rescheduling as possible: When trains are delayed, elaborate rescheduling is done. An example of rescheduling is depicted in Figure 6. Gray lines mean planned schedule (left). Let us assume that Train 1 is delayed at Station $\mathrm{Z}$ for some reason. A common method of modifying the schedule in this case is shown in black lines (right). The train-set of Train 1 is to be turned back as a deadhead train and stored in the depot located next to Station Y. Another train-set is urgently set up from the depot and driven to Station $X$ and it is assigned to Train 2 so that Train 2 can depart on time. This may be thought to be easy, but that is not true. Usually, there 
are no spare train-set at the depot. So, a lot of subsequent changes in trainset utilization schedule have to be made. In addition, there are no reserve crew as well. So, again a lot of subsequent changes in crew shift schedule have to be made. Figure 5 is just a simple example and in case of disruption, very elaborate rescheduling is done to reduce dissatisfaction of passengers as much as possible.

2. As standard as possible: Standard patterns and rules of rescheduling are prepared: In the case of the Yamagata and Akita Shinkansens, there occurs a serious problem about delay management if trains are delayed in conventional railway areas. Dispatchers have to make a decision whether they keep coupling or give up coupling at the junction station. JR East prepares a manual for such cases (see Figure 6). It describes if the delay is less than a certain threshold $\mathrm{x}$, coupling has to be done, meaning trains in the Tohoku Shinkansen (main line) have to wait. If the delay is larger than $\mathrm{x}$ and less than another threshold $\mathrm{y}$, coupling is given up (Figure 7 right). This means trains of the Akita/Yamagata Shinkansen run in the Tohoku Shinkansen line alone. If the delay is larger than y, the train is coupled with the next train (Train 2 in Figure 7) of the Tohoku Shinkansen, because it is very likely that the next train (Train 12) is also delayed. In case of giving up coupling, the problem is again in assigning crew. One more crew has to be squeezed out by drastically changing the subsequent crew schedules.

3. As simple as possible: This could be considered to be an important and basic idea in the operation of railways in Japan. I would say that in Japan it is believed that usage of facilities must be as simple as possible. There is an idea in the background that if usage of facilities is complicated, it might cause operational errors because there are so many trains running.

For example, "double single track" is seldom used in Japan. This is because people hate to complicate usage of tracks, which is crucial in guaranteeing safety.

Another example is the drivers' shift. Licences for the Shinkansen are different from those of conventional railway lines. This means a driver of conventional railway lines is not permitted to drive the Shinkansen (and vice
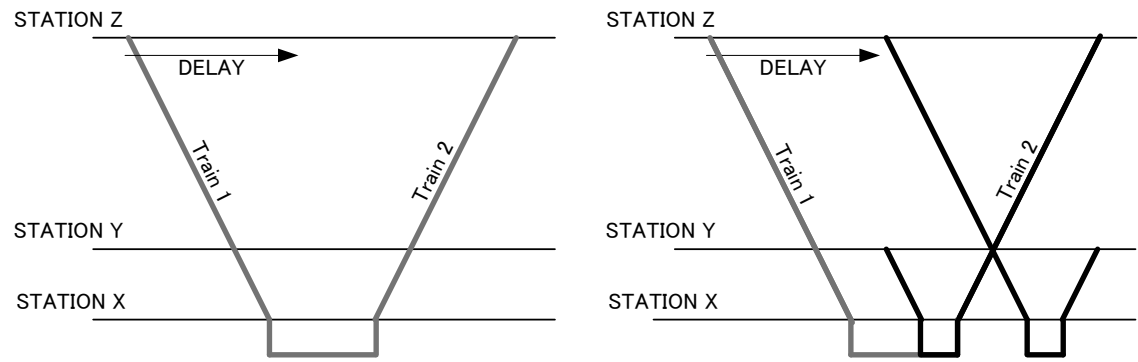

Figure 6: An example of rescheduling. 


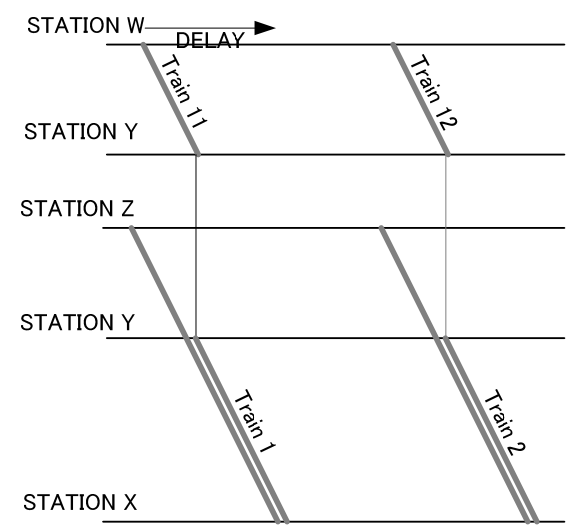

(a) Planned

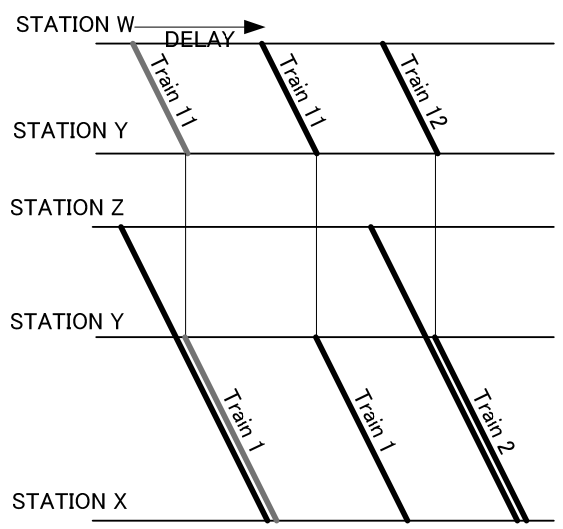

(b) Result

Figure 7: Delay management at a junction station where trains are coupled.

versa). Hence, it is impossible for a driver to drive a Shinkansen train and a conventional railway line train on the same day. This is to avoid drivers make any mistakes in operation because equipments of train-sets and operational rules are different between the Shinkansen and conventional railway lines.

One more example is seat layouts of Shinkansen trains. Series 700 and Series N700 Shinkansen train-sets of the Tokaido Shinkansen were designed so that the seat layouts are totally the same as Series 300, which was mainly used at that time. This is because even if a Series 300 train-set is suddenly substituted with a Series 700 or N700 (or vice versa), it is not necessary to give any further announcement to passengers for their new seats, and it is all right just to say "Please take the seat described on your ticket." Otherwise, extensive guidance to passengers about their new seats becomes necessary.

It might be true that by making usage of facilities complicated, you can maximize the performance of the facilities. For example, in case of the double single track, if a train has an engine trouble between stations, you can continue train operation using the other track, which is not possible in a double track line. So, to cope with such a criticism, a lot of efforts to increase reliability of hardware are made in Japan in compensation of making usage of facilities simple.

4. As much training as possible: Route settings of all trains for all stations are automatically done by the programmed route control system (PRC). PRC has a long history and has an extremely high reliability. However, in case of a system-down, training of manual route setting is always done. It may be improbable that this training is of any use one day, but they continue the training just for a rainy day.

5. As much efforts as possible: As described in the previous section, in some part of the Tokaido Shinkansen line, they sometimes have a lot of snow in 
winter. The worst record was made in 1976. 635 trains were cancelled in that year due to heavy snow and the average delay of trains on snowy days was 20.1 minutes. Running speed must be decreased to avoid catching the snow and the snow on the surface of train-sets has to be removed. This means that trains are very much delayed on snowy days. In former days, people hit the snow on the train-set with a wooden stick to fall it down. Obviously, it took a lot of time and labour. Now, a new equipment using high pressure is used to remove the snow. More than that, JR Central made an intensive research about effective countermeasures to reduce damages caused by snow such as to install sprinklers to make snow wet and monitoring system for the condition of snow to decide when the sprinkler should be started and so on [5]. The result was outstanding. For more than 15 years, no trains were cancelled due to snow and the average delays on snowy days in fiscal years 2006 and 2007 were 1.4 minutes and 1.7 minutes respectively.

\section{Conclusions}

The Shinkansen of Japan is well known for its punctuality. Although there are not abundant resources available, various kinds of ideas and hard training of dispatchers and crews have realized the punctuality.

The Tohoku Shinkansen is extended to Aomori this year. The Kyushu Shinkansen between Hakata and Yatsushiro is opened next year and it is planned that some trains go to Kagoshima directly from Osaka. The lines and timetables of Shinkansens become more and more complicated but I am quite sure that this punctuality level will be kept in the future.

\section{References}

[1] JR East Annual Report 2009: http://www.jreast.co.jp/e/investor/ar/2009/

[2] Annual Report of JR Central (in Japanese), 2009.

[3] Facts and Figures of Japanese Railways - 2009 (in Japanese), Institution for Transport Policy Studies, 2009.

[4] Environmental Report of JR Central 2009: http://english.jrcentral.co.jp/company/company/others/eco-report/_pdf/kankyo2009-e.pdf.

[5] T. Amatani: Countermeasures to reduce delays of trains on snowy days (in Japanese), Technical Report of JR Central, Vol.8, 2009. 